\title{
Mejoras en los procesos logísticos del centro de distribución y almacenaje de alimentos
}

\section{Improvements in the logistics processes of the food storage and distribution center}

\author{
Osnaider Ávila, osnaideravila@gmail.com \\ Milagros Thielen González, thilin 22@hotmail.com \\ Universidad José Antonio Páez, Venezuela
}

\section{RESUMEN}

La presente investigación tuvo como propósito el desarrollo de estrategias necesarias en el mejoramiento y ampliación de los almacenes, para observar un avance eficaz en los procesos de producción y distribución de la empresa Distribuidora J.S, ubicada en Maracay, Estado Aragua. Se consideró una población de diez (10) trabajadores que laboraban en las áreas del almacén de materia prima, con el objetivo de obtener información con datos fiables que sirvieron de base para el desarrollo de la investigación. Se desplegaron cuatro (4) fases de investigación. La primera consistió en el diagnóstico de la situación en cuanto al sistema de operaciones; en la segunda fase, se analizó los factores que inciden en el área de almacén a través de una lluvia de ideas, para detectar la causa raíz del problema. En la tercera fase se diseñó una propuesta del sistema logístico, utilizando herramientas de mejora como la metodología 5S y clasificación ABC. Finalmente, se estudió la relación costo-beneficio.

Palabras clave: Almacén; inventario; procesos logísticos, producción y distribución

The purpose of this research was the development of necessary strategies for the improvement and expansion of warehouses, to observe an effective advance in the production and distribution processes of the Distribuidora J.S company, located in Maracay, Aragua State. A population of ten (10) workers who worked in the areas of the raw material warehouse was considered, with the objective of obtaining information with reliable data that served as the basis for the development of the research. Four (4) research phases were deployed. The first consisted of the diagnosis of the situation regarding the operating system; In the second phase, the factors that affect the warehouse area were analyzed through a brainstorm to detect the root cause of the problem. In the third phase, a proposal of the logistics system was designed, using improvement tools such as the $5 \mathrm{~S}$ methodology and $\mathrm{ABC}$ classification. Finally, the cost-benefit ratio was studied.

Key words: Warehouse; Inventory; logistics processes, production and distribution 
INTRODUCCIÓN

La logística es una de las actividades de relevancia en el contexto de las empresas del siglo XXI, que buscan por todos los medios maximizar el nivel de satisfacción del cliente, a través de la distribución de productos con altos estándares de calidad, con un servicio esmerado y entregados en el tiempo oportuno (Tejero, 2007) (De Pablos, Agius, Romero, y Salgado, 2017). Los modelos administrativos ofrecen una solución duradera para los problemas que atraviesan las empresas en el área de logística y abastecimiento (Pierre y Sánchez, 2017), por esto la presente investigación se involucra en estas actividades de la cadena de valor para maximizar el nivel de satisfacción de los clientes.

La logística busca gerenciar estratégicamente la adquisición, el movimiento, el almacenamiento de productos y el control de inventarios, así como todo el flujo de información asociado, a través de los cuales la organización y su canal de distribución se encauzan de modo tal que la rentabilidad presente y futura de la empresa es maximizada en términos de costos $y$ efectividad (Briñez y Torres, 2015). Anteriormente, la logística era sólo tener el producto justo, en el sitio justo, en el tiempo oportuno, al menor costo posible (Riviera, Delagado, y Carrillo, 2013). Actualmente, estas actividades aparentemente sencillas han sido redefinidas y ahora son todo un proceso (Ramírez, 2009).

La cadena de suministro es una de las herramientas de mayor uso en el campo de la ingeniería (García, 2016) , debido a que a través del análisis de cada una de las etapas de la producción se pueden identificar las debilidades en los procesos y propiciar decisiones óptimas para su solución. Donde se involucra a todas las partes interesadas, que incluyen a proveedores, clientes, personal de la organización, competidores, instituciones del estado y la comunidad que se encuentre inmersa en el entorno empresarial.

El almacén es un lugar especialmente estructurado y planificado para custodiar, proteger y controlar los bienes de activo fijo, que se consideran redituable para una empresa (Céspedes, Aliaga, Pérez, Díaz, y Figueredo, 2017) (Estupiñán, 2017). Es importante hacer hincapié en que lo almacenado debe tener un movimiento rápido de entrada y salida, es decir, una rápida rotación. Todo manejo, almacenamiento de materiales $y$ productos es algo que eleva el costo final sin agregarle valor. La Distribuidora J.S cuenta con un (1) almacén de materia prima y un (1) almacén de producto terminado. En se almacena harinas leudantes y polvo de hornear, entres otros, y así satisfacer el mercado de panificadoras y panaderías. En los últimos 3 años la Empresa ha tenido un crecimiento acelerado, que se ve reflejado en el incremento de los volúmenes de venta en un $25 \%$ y, por lo tanto, ha llevado a adquirir mayor cantidad de materia prima, que aumentó a 300 unidades de inventario (stock keeping unit) para satisfacer el mismo. Por tal motivo se hizo necesario la ampliación y adquisición de nuevos equipos para progresivamente regularizar el proceso logístico de la empresa.

Los almacenes se han visto seriamente afectados por los siguientes factores: capacidad limitada de almacenamiento, mala distribución en los almacenes de materia prima y un conteo manual propenso a errores humanos, ocasionando pérdidas en los inventarios y en los costos directos e indirectos 
considerando lo expuesto Distribuidora $J . S$, no ha logrado superar en su almacén de materia prima los índices de confiabilidad de inventarios más allá del $40 \%$ en los últimos dos (2) años, estando su último inventario alrededor de un $17 \%$ de confiabilidad general, de acuerdo de los indicadores de gestión manejados por la empresa. En este sentido, se presenta un plan de mejora en los procesos logísticos de la empresa, donde se evaluaron los procesos llevados a cabo en el almacén de materia prima, desde la recepción de los insumos hasta su despacho al área de manufactura, pasando por todo el control y gestión del inventario.

\section{MATERIALES Y MÉTODO}

La metodología fue dividida en cinco (5) fases, las cuales se describen a continuación:

\section{Fase I: Diagnosticar la situación actual de los procesos logísticos}

En esta fase se comienza con el diagnóstico de la situación actual del ciclo logístico del almacén, es decir, la forma como realizan las actividades en el área de recepción hasta despacho interno y terceros. Estableciendo comparaciones con respecto a la teoría de inventario permitiendo determinar situaciones de posibles problemas dentro del ciclo logístico que se lleve a cabo actualmente. Para este diagnóstico la información necesaria se recopila mediante la aplicación de la entrevista no estructurada (Díaz-Bravo, Torruco-García, MartínesHernandez, y Valera-Ruíz, 2013) al personal involucrado con el área de interés. De la misma forma se realiza una tormenta de ideas (González, 2008) conjuntamente con el personal obteniendo así una mejor percepción del sistema.

También se evaluó la situación interna y externa de la empresa mediante un análisis FODA (Ramirez, 2017) y así mismo se hizo necesario la revisión de todos los procedimientos operativos $\mathrm{y}$ administrativos, condiciones de trabajo, funciones del personal y verificación continúa de las disponibilidades de los insumos de acuerdo a los requerimientos de planificación y manufactura.

\section{Fase II: Analizar las problemáticas detectadas en la fase de diagnóstico}

Para el desarrollo de esta fase se aplica la técnica de grupo nominal y se jerarquizaron las causas de acuerdo a su importancia conjuntamente con el personal que labora en el almacén. Con esta herramienta se pudo evaluar en cuanto a porcentajes cuales son los problemas que tienen mayor incidencia dentro del proceso logístico, en conjunto con un diagrama de Pareto (Yanes y Gaitan, 2005).

\section{Fase III: Diseñar el plan de mejoras en los procesos logísticos}

El objetivo de esta fase es proponer un plan de acción que permita mejorar la confiabilidad del inventario de materia prima. Para esto se desarrollara un sistema de control y mantenimiento continuo del inventario (Viloria, 2005) donde debe haber la participación y compromiso de todo el personal que labore en el almacén, desde montacarguistas hasta la Gerencia, utilizando herramientas de mejoras continuas como las 5S (Velasco, 2016) (Bruque, 2014) la cual agrupa una serie de actividades que se desarrollan con el objetivo de crear condiciones de trabajo que permitan la ejecución de labores de forma organizada, ordenada y limpia. Una nueva distribución de los productos en el almacén y por último la aplicación de un taller de capacitación a los trabajadores para con la intención de que los mismos 
reconozcan todo lo relacionado con la logística y distribución de la empresa.

\section{Fase IV, Analizar la relación costo- beneficio}

En esta fase se debe tomar en consideración todos los costos operacionales, materiales y técnicos presentes en la propuesta elaborada, con la finalidad de compararlos con sus beneficios tangibles e intangibles. Posteriormente, se representar gráficamente el tiempo de retorno de la inversión realizada, concluyendo así, si el proyecto es factible o no de llevarlo a cabo. El análisis de costo-beneficio (Mejía y de Desarrollo, 2012) es una técnica importante dentro del ámbito de la teoría de la decisión (Vitoriano, 2007) (Bonatti, 2011). Pretende determinar la conveniencia de un proyecto mediante la enumeración y valoración posterior en términos monetarios de todos los costos y beneficios derivados directa e indirecta de dicho proyecto.

\section{RESULTADO Y DISCUSIÓN}

Fase I: Diagnosticar la situación actual de los procesos logísticos

\section{Descripción general del almacén}

- Cuenta con un área de aproximadamente $500 \mathrm{~m}^{2}$ a cual está dividida en: almacén de mercancías, área de recepción y despacho, área administrativa y los sanitarios.

- Todas las paredes de la planta están cubiertas con pintura aducto-amina food grade $100 \%$ sólido, los pisos son de cemento tratado absorbente, liso y lavable. Estos cuentan con un sistema de drenaje que evita la acumulación de agua y polvo, los cuales se mantiene limpios y secos durante las actividades de producción.
- El local está diseñado para impedir la entrada de aves y otras plagas.

- El ambiente está aislado del entorno con controles de acceso y flujo lógico de personas, materiales y producto terminado.

\section{Normas básicas de almacén}

- Las zonas de almacenamiento debe estar perfectamente delimitado $\mathrm{y}$ señalizado, no se puede almacenar fuera de ellas.

- Los pasillos deben ser rectos y conducir directamente a las salidas

- Debe evitarse que los envases, embalajes o cualquier tipo de objeto impidan la visualización de señalización y bloqueen las salidas de evacuación (Emergencias).

- El almacén debe tener fácil acceso a los extintores de incendio y salidas para el personal libres de obstáculos.

- Almacenar según sea el producto o material.

- Evitar accidentes que puedan llegar hacer graves, y que no afecten las instalaciones y los productos almacenados.

\section{Proceso de recepción de mercancía}

El proceso de recepción cuenta con lo siguiente:

1. El proveedor entrega la factura al encargado del almacén.

2. Se chequea cantidad, tipo de mercancía.

3. Si todo está en orden se lleva la factura a la oficina de compras para la tramitación de pagos.

4. Si hay algún faltante de mercancía se hace la observación para posteriormente llamar al proveedor.

5. Se da la orden de descargar la mercancía. 


\section{Proceso del almacenaje de mercancía}

El proceso de almacenamiento cuenta con lo siguiente:

1. Una vez descargado el camión los almacenistas ubican un espacio disponible para la colocación de la mercancía.

2. La mercancía es trasladada en un montacargas por los almacenistas hasta el sitio donde será almacenada.

3. Se procede a la impresión de las etiquetas que contienen los códigos para luego ser etiquetada la mercancía.

4. Una vez procesada la factura y debidamente identificada la mercancía, se da la orden para abastecer los racks en la mercancía faltante. Esta actividad se realiza verificando faltantes de mercancías en los racks correspondientes y trasladándose hasta el almacén para realizar su búsqueda.

\section{Proceso de despacho de mercancía}

$\mathrm{Al}$ momento que se recibe la solicitud de mercancía se procede a realizar el despacho del pedido, este proceso cuenta con los siguientes pasos:

1. Al momento de preparar el pedido de mercancía, se verifica la fecha de ingreso al almacén, corroborando que se le de salida a la mercancía que tiene más tiempo en el almacén, según la fecha de recepción.

2. La mercancía debe ser tomada del área de almacenamiento, garantizando que no se de apertura a una caja o empaque, mientras haya otra en suministro.

3. Una vez listo el pedido, se lleva al área de entrega, indicando la verificación en cantidades y calidad. La mercancía es colocada en el lugar asignado a despachos para ser retirada por la persona encargada de su entrega al cliente.

\section{Observación directa}

A través de la lista de verificación se puede determinar que el proceso logístico cuenta con un control e inspección en la recepción de mercancía regular, debido a que al instante de recibirla en ocasiones no es ingresada inmediatamente. Por lo tanto, no se verifica por completo, esto conlleva a un inadecuado manejo de la mercancía que genera pérdida. Por otra parte, las operaciones no cuentan con formatos normalizados que dejen por sentado las actividades que se deban realizar correctamente, ni se encuentran regidos por un procedimiento que determine los pasos a seguir para mantener un mejor control. Es por ello, que los almacenistas omiten operaciones o procedimientos y realizan en algunas oportunidades actividades de acuerdo a sus interpretaciones, lo que puede traer como consecuencia irregularidades en la administración del inventario de mercancías. Los procedimientos garantizan a la empresa, como lograr la satisfacción para llegar a obtener un nivel óptimo de eficiencia. Al no poseer las normas y procedimientos para el manejo de control de inventario, la organización no puede alcanzar un control interno adecuado. Al contrario, para lograr esto, se requiere comunicación y monitoreo continuo sobre la ejecución de las normas.

No cuenta con un control, detallado de entradas y salidas de materiales ni con un conteo actualizado de los mismo, por lo que la persona encargada de las compras simplemente se comunica vía telefónica con el proveedor para que le suministre los artículos, en muchos casos innecesarios, por no existir un seguimiento formal y un control adecuado para determinar la falta o no de los materiales. En cuanto al almacenamiento de la mercancía, esta no es la más adecuada, en muchas ocasiones se encuentran 
mercancías que no están ubicados en el estante correcto, generando malestar tanto al trabajador como al cliente por el tiempo de espera que le causa.

\section{Resultados de la entrevista no estructurada}

Con la ejecución de la entrevista no estructurada aplicada a los trabajadores que labora de forma directa en el almacén de la organización, se estableció que las fallas existentes en el área según lo manifestaron los almacenista, quienes consideran que algunas de las causas que están produciendo dicha problemáticas son por la falta de aplicación de procedimientos estandarizados y mala distribución de los materiales, así como también, a la ausencia de una óptima logística de las diligencias que se ejecutan para el manejo y control de materiales. Por otra parte, los trabajadores reportaron la existencia de fallas que en la actualidad están afectando el desenvolvimiento de las actividades requeridas para el proceso de almacenamiento en la empresa en la que se puede mencionar:

1. Falta de visualización en el rayado del almacén.

2. Inadecuada utilización de los espacios en el almacén.

3. Obstaculización por presencia de desperdicios en el área de trabajo.

4. Ausencia de supervisión de las actividades ejecutadas.

5. Falta de identificación de las materias primas.

6. Materiales entre mezclados (materia prima-producto terminado)

\section{Análisis FODA}

A través del análisis FODA se evalúa la situación interna respecto a la disponibilidad de recursos de capital, personal, activos, calidad de producto, estructura interna y de mercado, percepción de los consumidores, entre otros y la situación externa en cuanto oportunidades y amenazas que el contexto puede 44 presentarle a Distribuidora J.S, Estudiando aspectos importantes en cuanto a los procesos logísticos de las organizaciones.

\section{a) Fortalezas}

Es de importancia tener instalaciones propias para cualquier organización, ya que permite hacer mejores inversiones en pro de los avances en la empresa y bajan los egresos en cuanto al alquiler de una instalación. En este sentido, la situación que está viviendo el país es vital ofrecer transporte y flete para los despachos de mercancía. Esto genera más confiabilidad y lealtad a la empresa por parte de sus clientes. Además, ofrecer buenos precios y que el producto sea de la mejor calidad para la satisfacción de los consumidores es un factor importante además de crear preferencia a favor de la organización.

\section{b) Debilidades}

La empresa tiene un sistema de registro deficiente, en cuanto a la elaboración de sus actividades, por lo que se ve afectada para manejar información necesaria en cuanto a la recepción, compras. y diferentes procesos realizados con anterioridad. De la misma manera presenta fallas en cuanto al manejo de sus proveedores por e incumplimiento de pedidos de materia prima lo cual genera retrasos en el proceso. También presentan déficit en cuanto a la planeación, seguimiento y control de las actividades ocasionando mucho más retrasos en el desarrollo logístico dentro del almacén.

\section{c) Oportunidades}

Aumentar el número de clientes con los que cuenta la empresa en cuanto la expansión del mercado, cada vez hay más 
pequeñas y medias empresas interesadas en incursionar en el mundo de la panificación y es de gran importancia la captación de nuevos clientes que eleven las ventas y que promocionen el nombre de la empresa. Capacitar el personal a medida de los avances obtenidos representa una ayuda colectiva y comprometerse a ser mejores cada día para obtener las metas trazadas.

\section{d) Amenazas}

La situación país es la mayor amenaza que enfrentan la mayoría de las organizaciones hoy en día y más las que se ubican en el sector alimentario. Además de esto esta única razón con lleva al desate de la delincuencia a mayor escala y un desabastecimiento de materia prima que impacta de forma importante los inventarios del almacén.

\section{Fallas encontradas en el proceso}

Luego de diagnosticar la situación actual a través de la observación directa y de la información obtenida por parte de los empleados en la entrevista no estructurada pudimos detectar algunas las posibles causas que originan los diferentes problemas como lo son:

- Mal almacenamiento

- Falta de identificación

- Fallas en la clasificación

- Despidos justificados

- Falta de capacitación

- Poca comunicación

- Desorganización

- Inutilización de los espacios

- Procedimientos no adecuados

- Medio Ambiente

- Poca ventilación

- Iluminación deficiente
- Falta de orden y limpieza

- Maquinarias y equipos

- Falta de Racks

- Falta de mantenimiento en los equipos

\section{Fase II: Analizar las problemáticas detectadas en la fase de diagnóstico}

En la presente fase se lleva a cabo un análisis del proceso logístico que se realiza en la empresa Distribuidora JS C.A, a través de la aplicación de una serie de herramientas de ingeniería industrial que permita identificar las actividades improductivas presentes y sus causas.

\section{Diagrama causa-efecto}

Se construyó un diagrama muestra el diagrama causa-efecto donde se representa cada una de las fallas detectadas en la fase del diagnóstico, las cuales son causantes de los problemas en el proceso y se encuentran en métodos, mano de obra, medio ambiente, materiales y maquinaria y equipos. En este diagrama podemos resaltar que los factores de mayor influencia en la baja confiabilidad del proceso logístico en la empresa son los procedimientos de trabajo acompañado y la poca comunicación entre el personal en sus diferentes áreas. Esta situación no permite el buen desarrollo de las actividades, tal cual como debe gestionarse un almacén de materia prima.

A través de la tormenta de ideas se obtuvo la información del personal involucrado en la gestión del proceso, expresando sus ideas sobre las posibles causas que originan el problema. Los resultados de la tormenta de ideas son ordenandos y mostrados en un diagrama causa-efecto. 
Del diagrama de causa-efecto se puede resaltar lo siguiente

\section{Materiales:}

- Mal almacenamiento: No se hace un buen uso en las zonas dentro del almacén, el cual conlleva al desaprovechamiento de altura y espacios en los racks.

- Falta de identificación: La mayoría de la mercancía no se encuentra bien identificada o simplemente no tiene identificación de ningún tipo lo que trae consigo confusión al momento de seleccionar la mercancía para su despacho y utilización.

- Fallas en la clasificación: La falta de clasificación en la mercancía origina pérdida de tiempo para la búsqueda de la misma y mucha confusión a la hora de buscar algún tipo de producto

\section{Mano de obra}

- Despidos justificados: Debido a la situación actual se han registrado robos de mercancía efectuados por los trabajadores en el área de almacén, ocasionando su despido inmediato. Esto conlleva a nuevas contrataciones, nuevos procesos de inducción y capacitación, retrasando y desmejorando los procesos operativos de la empresa.

- Falta de capacitación: los trabajadores en el área de almacén requieren un entrenamiento en cuanto al manejo de materiales, identificación y almacenamiento de materias primas.

- Poca comunicación: La falta de comunicación entre los trabajadores en cuanto a las tareas realizadas y las no realizadas afectan seriamente en transcurso del proceso, ya que no hay un seguimiento.

\section{e) Métodos:}

- Desorganización: Al momento de ubicar mercancía nueva en el almacén los trabajadores las colocan en lugares disponibles, esto conlleva a que las mercancías se mezclen entre sí y estén dispersas por todo el almacén.

- Inutilización de los espacios: Dentro del almacén se encuentran diferentes espacios que podrían ser indicados para la colocación de nueva estantería y en su defecto estos servirían para tener mayor organización y más espacio para la colocación de mercancía, sin embargo, estos espacio se encuentran ocupados por equipos y objetos innecesarios.

- Procedimientos no adecuados: una de las actividades fundamentales dentro del almacén y que debe estar enfocada para cumplirse al $100 \%$ de los requerimientos del cliente es el picking. Actualmente se tiene un procedimiento que está generando retrabajo debido a que el mismo es realizado con la solicitud de facturación y por lo general en la mayoría de los casos no es igual a la factura despachada, por lo que se debe realizar un nuevo chequeo del pedido $y$ adicionar $o$ retirar mercancía.

\section{Medio Ambiente}

- Poca ventilación: en el almacén la ventilación que se genera es la que ingresa por las diferentes entradas y portones, sin embargo, esta no es suficiente como para ventilar cada una de las áreas con las que el almacén cuenta.

- Iluminación deficiente: La falta de iluminación que presenta el almacén 
- genera que la jornada laboral se encuentre pesada, creando fatiga y cansancio visual en los trabajadores.

- Falta de orden y limpieza: Se pudo determinar que la principal causa es que en el almacén predomina el desorden, no hay lugares específicos para el almacenamiento, las zonas no están bien definidas, no existe una identificación (pasillos, productos, entre otros), no existe una rotación de material ocasionando pérdidas mayores por deterioro. De igual manera se encontró la presencia de desperdicios y basura en los alrededores.

\section{Maquinarias y equipos}

- Falta de Racks: En la actualidad no existen la cantidad necesaria de racks y estantería requerida para almacenar toda la mercancía requerida dentro del almacén.

- Falta de mantenimiento en los equipos: La empresa actualmente tienen un montacargas que no está en funcionamiento ya que no tiene un plan de mantenimiento.

\section{Técnica del grupo nominal}

Partiendo de la información obtenida en la fase del diagnóstico, tomando como referencia los resultados arrojados en el diagrama de causa-efecto se procede a jerarquizar los problemas a través de la técnica del grupo nominal, la cual consiste en una votación realizada por diez (10) trabajadores involucrados directamente en el proceso logístico, considerando una calificación de uno (1) a cinco (5) puntos. Así podrán evaluar las cinco (5) causas más importantes según su criterio, teniendo en cuenta que uno (1) es la menos grave y cinco es el valor de mayor gravedad. Con esto los trabajadores pueden calificar cuales de los problemas son los que están ocasionando la deficiencia con mayor incidencia en el proceso logístico y el resto de las situaciones en el almacén

En el cuadro anterior se puede observar que las primeras nueve causas representan el $80 \%$ de los problemas observados en el proceso logístico. Una vez culminadas la aplicación de las técnicas que han ayudado al arrojamiento de datos.

\section{Resumen de oportunidades de mejoras encontradas}

Después de haber realizado el estudio de las principales causas que generan los problemas en el proceso logístico, se analizó cada una de ellas con el personal involucrado en el proceso y mediante una tormenta de ideas, aportar oportunidades de mejoras y crear un plan de acción que permita resolver las fallas presentes en el desarrollo de las actividades. A continuación se muestra un resumen de las oportunidades de mejora presentes partiendo de las fallas más notables:

- Falta de Capacitación: Elaborar programas de capacitación para el personal e involucrar a cada uno de ellos escuchando sus necesidades y tomando en cuenta el aporte de sus ideas, además de aumentar las buenas prácticas en el sitio de trabajo mediante la aplicación de charlas de seguridad, higiene $y$ manejo de materiales.

- Desorganización: Realizar un plan de acción con diferentes estrategias que permitan llevar un control y un buen seguimiento en cuanto a todas las actividades involucradas en el proceso logístico, pues así se puede garantizar un mejor desenvolvimiento en las actividades diarias. 
- Fallas en la clasificación de mercancía: Elaborar un control en cuanto a la entrada de mercancía al almacén bajo la realización de etiquetas de clasificación por categorías debidamente especificadas. Además de llevar un registro en cuanto la colocación y ubicación de cada una de estas etiquetas.

- Falta de orden y limpieza: Planificar y establecer jornadas de limpieza en periodos estipulados bajo supervisión y verificación del personal encargado. Esto ayudara a mantener el almacén más limpio y ordenado además de concientizar a todo el personal que labora en el almacén para mantener las áreas y el trabajo realizado por las personas de mantenimiento.

\section{Fase III: Diseñar el plan de mejoras en los procesos logísticos}

La elaboración de este diseño sirve como base para la posterior realización de un manual de procedimiento, que proporcionará ventajas inmediatas, tales como: mantener un mejor control del inventario, mantener el stock de materiales actualizado, al igual que permitir tener conocimiento de la existencia física de los movimientos realizados para un tiempo determinado. Para la optimización de la gestión de almacenamiento es necesario considerar diversos aspectos tales como: condiciones del almacén; distribución; recepción, clasificación, iluminación, ventilación, despacho e inventario de materiales.

Las acciones a seguir fueron determinadas luego de diagnosticar la problemática en el área, un aspecto a atender es el espacio físico, es un factor importante en todo almacén, para esto se consideró el volumen de operaciones diarias, esto debe ser atendido por los departamentos de planificación y almacén.
Seguidamente se determinó el problema que presenta el actual procedimiento para la toma de muestras por parte del departamento de calidad, se debe modificar el procedimiento de forma que no presente alteraciones considerables a los principios básicos del muestreo; por último se consideró que es necesario conjuntamente con el departamento de sistemas realizar los ajustes necesario al sistema operativo y mejorar la gestión de almacén.

Debido a la problemática $\mathrm{y}$ detectando las oportunidades de mejora se inicia la propuesta mediante la elaboración de un plan de acción como instrumento de programación y control en la ejecución de las actividades definidas para mejorar la gestión del almacén de materia prima y aumentar la confiabilidad del inventario.

\section{Distribución inadecuada del almacén de mercancías}

Actualmente el almacén se encuentra desorganizado lo que ocasiona que los materiales se almacenan inadecuadamente. Por esta razón se propone aplicar la teoría de las 5S, estas actividades se expresan a continuación:

- Seiri (Clasificar): esta actividad consiste en retirar y eliminar los elementos innecesarios para las operaciones. En este caso se clasifican los materiales obsoletos y en mal estado para que la empresa disponga de ellos.

- El proceso de selección que se debe seguir para remover los artículos innecesarios del área de trabajo son:

- Reconocer el área de oportunidad: Esta lista ayuda a detectar áreas u objetos que por su naturaleza pudieran pasar desapercibido.

- Definir los criterios de selección: Es importante definir un estándar que 
ayude a diferenciar lo que es realmente necesario de lo que no lo es.

- Identificar los objetos seleccionados: los objetos seleccionados como no necesarios deben ser identificados y ser llevados al área de recuperación o destrucción de ser el caso, los mismos son trasladados con los Traspaletas o montacargas dependiendo de las cantidades.

- Evaluar los objetos seleccionados: En esta etapa los responsables del área deciden qué hacer con los objetos que fueron seleccionados como no necesarios en este caso el encarado directo el supervisor de almacén.

\section{CONCLUSIONES}

A través de los indicadores de gestión se espera medir el desempeño de varios procesos que se llevan a cabo dentro del área logística con el fin de reducir errores y encontrar posibles soluciones para los mismos con el fin de dar respuesta inmediata para los requerimientos del cliente.

El diagnóstico realizado en el área de almacén de la empresa Distribuidora JS $C . A$., se efectúa con el fin de identificar los aspectos en donde se presentan deficiencias y posteriormente proponer un plan de mejoras que permita la optimización del proceso logístico.

Atendiendo las necesidades de la empresa, se aplicó un conjunto de herramientas de estudio que permitieron evaluar la situación actual para proponer un plan de mejoras de los procesos logísticos para la empresa Distribuidora JS C.A.; a través de la observación directa y del uso del diagrama Causa-Efecto, los inconvenientes que presenta dicho almacén para llevar a cabo una óptima gestión de almacenaje por medio de la aplicación de técnicas que garanticen la obtención de artículos de manera oportuna y en el peor de los casos el aprovechamiento del espacio para albergar todos los productos.

Los aspectos que se identificaron en la primera fase a través de las herramientas aplicadas dentro de los procesos de almacenamiento fue en la recepción de material; este proceso no se está realizando de la manera más correcta, ya que en el desarrollo de las actividades se están presentando una serie de inconvenientes dentro de los cuales podemos resaltar, falta de un instructivo, zona de recibo no demarcada, no contar con el personal suficiente para el descargue de las mercancías, entre otros.

Por otro lado, el almacenamiento de materiales fue uno de los puntos clave en donde se presentaron la mayoría de las fallas, ya que actualmente los materiales se ubican sin ningún tipo de criterio en cualquier lugar o espacio que se encuentre libre.

En la segunda fase se procedió a determinar las causas de los problemas existentes en el proceso logístico, desde su recepción hasta su almacenamiento y despacho, donde las obtuvimos a través del Diagrama causas- efecto en conjunto con una tormenta de ideas. Además, se utiliza la técnica de grupo nominal aplicada al personal directamente involucrado con el proceso $y$ posteriormente se graficó para la mejor visualización de los problemas identificados, tales como, fallas en la clasificación de los productos y la falta de identificación de la mercancía, también el mal almacenamiento de la mercancía recibida ya que no poseen un procedimiento estandarizado que les permita realizar de manera eficiente el proceso. 
REFERENCIAS

Bonatti, P. (2011). Teoría de decisión.

Briñez, M., y Torres, F. (2015). Actividades estratégicas en la gestión logística de las empresas del sector lácteo del estado Zulia. Sapienza Organizacional, $2(4), 31-48$

Bruque, C. (2014). Análisis del proceso logístico de Procisa Ecuador SA y propuesta de mejora para garantizar una buena planificación y control de equipos aplicando la metodología $5 S$. Guayaquil: Universidad de Guayaquil

Céspedes, A., Aliaga, Z., Pérez, A., Díaz, C., y Figueredo, Y. (2017). Sistema informático para el control de piezas y componentes de taller de trasnporte del partido comunista de Cuba en Las Tunas. Revista de Investigación en Tecnologías de la Información, 5(10), 4

De Pablos, C., Agius, J., Romero, S., y Salgado, S. (2017). Organización y transformación de los sistemas de información en la empresas. Editorial ESIC

Díaz-Bravo, L., Torruco-García, U., Martínes-Hernandez, M., y ValeraRuíz, M. (2013). La entrevista, recuerso flexible y dinámico. Investigación en educación médica, 2(7), 162-167

Estupiñán, M. (2017). Propuesta de política de control de activos fijos desde las necesidades de la Corporación Unificada Nacional. Bogotá: La Salle.

García, L. (2016). Gestión Logística Integral: las mejores prácticas en la cadena de abastecimiento. Ecoe Ediciones

González, M. (2008). Tormenta de ideas ¡Qué tontería más genial! ACIMED, 17(4), 4
Mejía, F., y de Desarrollo, B. (2012). Una introducción al análisis económico: costo, efectividad y costo-beneficio. Santiago

Pierre, S., y Sánchez, H. (2018). Propuesta de mejora del área de almacén en la empresa AGN industriales SAC a fin de mejorar su gestión. Lima: UIGV.

Ramírez, A. (2009). Manual de la gestión logística del transporte y distribución de mercanciás. Barranquilla: Universidad del Norte

Ramirez, J. (2017). Procedeimiento para la elaboración de un análisis FIDA como herramienta de planeación estratégica en las empresas. Veracruz: Universidad Veracruzana

Riviera, R., Delagado, L., y Carrillo, J. (2013). Logística de transporte y su desarrollo. Observato

Tejero, J. (2007). Logística integral: la gestión operativa de la empresas. ESIC

Velasco, J. (2016). Implementación de la metodología $5 S$ (organización y limpieza) en el área de producción: sección maquinado de la empresa Balsasud SA. Guayaquil: Universidad de Guayaquil

Viloria, N. (2005). Factores que inciden en el sistema de control interno de una organización. Actualidad Contable Faces, 11(8), 4

Vitoriano, B. (2007). Teoría de la decisión: decisión con incertidumbre, decisión multicriterio y teoría de juegos. Madrid: Universidad Complutense de Madrid.

Yanes, J., y Gaitan, O. (2005). Herramientas para la gestión energética empresarial. Scientia et technica, 29(3), 169-174 